\title{
Influência da adequação postural em cadeira de rodas na função respiratória de pacientes com distrofia muscular de Duchenne
}

\author{
Influence of wheelchair positioning aids on the respiratory function \\ of patients with Duchenne muscular dystrophy
}

Carolina Oliveira Rodini', Luanda André Collange 2 , Yara Juliano ${ }^{3}$, Claudia Santos Oliveira ${ }^{4}$, Alexandre Marini Isola ${ }^{5}$, Sabrina Bauléo Almeida ${ }^{6}$, Maria Harue Misao $^{7}$

RESUMO I O objetivo deste estudo foi avaliar a influência da adequação postural em cadeira de rodas na função respiratória de pacientes com distrofia muscular de Duchenne (DMD). Participaram 12 pacientes com diagnóstico de DMD e que possuíam cadeira de rodas adaptada com idade variando de 10 a 22 anos. Cada indivíduo foi avaliado na própria cadeira de rodas e em uma cadeira de rodas padrão, ou seja, sem reclinação ou tilt. As cadeiras dos participantes possuíam adaptações no encosto e no assento, confeccionados de acordo com as especificidades de cada paciente. A avaliação consistiu em mensurar o volume minuto (VM), volume corrente (VC), capacidade vital forçada (CVF), pressões inspiratória (PImax) e expiratória (PEmax) máximas e pico de fluxo expiratório (PFE). Para análise dos dados, foi utilizado o teste $t$ pareado, adotando-se o nível de significância de 0,05. As adaptações resultaram em melhores valores estatisticamente significativos de todos os parâmetros respiratórios: VM (8.963,3 e 10.762,5 mL/min; p=0,028), VC (319,1 e 433,6 mL; $p=0,005)$, CVF (1.476,3 e 1.850 mL; $\mathrm{p}=0,005)$, PImax (-41,2 e -51,2 $\left.\mathrm{CmH}_{2} \mathrm{O} ; \mathrm{p}=0,022\right)$, PEmax (29,6 e 36,7 $\left.\mathrm{cmH}_{2} \mathrm{O} ; \mathrm{p}=0,004\right)$ e PFE (162,1 e 185 L/min; $p=0,018$ ). Nossos resultados sugerem que a adequação postural em
ABSTRACT I The purpose of this study was to determine the influence of wheelchair positioning aids on the respiratory function of patients with Duchenne muscular dystrophy (DMD). Twelve non-ambulatory DMD patients, between 10 to 22 years of age, were evaluated. They were assessed in their adapted wheelchairs and in a standard wheelchair without tilt or reclining. The wheelchairs of the participants possessed adaptations in the backrest and the seat, made according to the specifics of each patient. Minute volume (MV), tidal volume (TV), forced vital capacity (FVC), maximum inspiratory (MIP) and expiratory pressures (MEP) and peak expiratory flow (PEF) were measured. For data analysis we used the paired t-test adopting the significance level of 0.05. The positioning aids resulted in statistically significant better values of all respiratory parameters: MV (8,963.3 and 10,762.5 mL/min; $p=0.028$ ), TV (319.1 and $433.6 \mathrm{~mL}$; $\mathrm{p}=0.005)$, FVC (1,476.3 and 1,850 $\mathrm{mL} ; \mathrm{p}=0.005)$, MIP (-41.2 and -51.2 $\mathrm{cmH}_{2} \mathrm{O} ; \mathrm{p}=0.022$ ), MEP (29.6 and $36.7 \mathrm{CmH}_{2} \mathrm{O} ; \mathrm{p}=0.004$ ) and PEF (162.1 and $185 \mathrm{~L} / \mathrm{min}$; $p=0.018)$. These results may suggest that wheelchair

\footnotetext{
Estudo desenvolvido na Associação de Assistência à Criança Deficiente (AACD) - São Paulo (SP), Brasil.

'Fisioterapeuta Especialista em Fisioterapia Neurológica no Setor de Fisioterapia Infantil da AACD; Mestranda pelo Departamento de Neurologia e Neurocirurgia da Universidade Federal de São Paulo (UNIFESP) - São Paulo (SP), Brasil.

2Fisioterapeuta Especialista em Fisioterapia Respiratória e Neurológica no Setor de Fisioterapia Infantil da AACD; Mestranda em Ciências da Reabilitação da Universidade Nove de Julho - São Paulo (SP), Brasil.

${ }^{3}$ Professora Titular da Disciplina de Saúde Coletiva da Faculdade de Medicina da Universidade de Santo Amaro (UNISA) - São Paulo (SP), Brasil.

${ }^{4}$ Fisioterapeuta; Professora do Curso de Mestrado e Doutorado em Ciências da Reabilitação da Universidade Nove de Julho - São Paulo (SP), Brasil.

${ }^{5}$ Médico Especialista em Pneumologia e Terapia intensiva; Pneumologista da AACD - São Paulo (SP), Brasil.

${ }^{6}$ Fisioterapeuta Especialista em Neurologia e Tecnologia Assistiva no Setor de Fisioterapia Infantil da AACD - São Paulo (SP), Brasil.

${ }^{7}$ Fisioterapeuta Especialista em Fisioterapia Respiratória e Neurológica no Setor de Fisioterapia Infantil da AACD - São Paulo (SP), Brasil.
} 
cadeira de rodas influenciou positivamente a função respiratória de pacientes com DMD.

Descritores | distrofia muscular de Duchenne; cadeiras de rodas; sistema respiratório; postura. positioning aids can positively influence pulmonary function for non-ambulatory DMD patients.

Keywords I Duchenne muscular dystrophy; wheelchairs; respiratory system; posture.

\section{INTRODUÇÃO}

A distrofia muscular de Duchenne (DMD), com incidência de 1/3.500 meninos nascidos vivos ${ }^{1}$, é uma desordem neuromuscular hereditária ligada ao cromossomo $\mathrm{X}^{2}$, que cursa com perda progressiva de força muscular e ocorre devido à mutação do gene da distrofina. A doença envolve predominantemente a musculatura proximal ${ }^{3}$, sendo que os sintomas se iniciam antes dos cinco anos de idade. Com a evolução da doença, a perda progressiva de força faz o indivíduo parar de andar por volta dos 9 aos 12 anos, além de levar ao desenvolvimento de escoliose grave ${ }^{4}$.

O prognóstico em longo prazo em DMD é determinado principalmente pela deterioração respiratória ${ }^{5}$ que tende a ser previsível e resulta da fraqueza dos músculos respiratórios, torando-se evidente quando o paciente deixa de andar de modo independente 6 . $\mathrm{O}$ acometimento pulmonar restritivo progressivo se inicia em torno de 10 a 12 anos de idade ${ }^{7}$ e tem sido associado com maior risco de complicações respiratórias e morte, de acordo com a extensão em que a função pulmonar e trocas gasosas estão comprometidas ${ }^{8,9}$. A perda de força na musculatura inspiratória e expiratória seguida por tosse ineficaz e diminuição de ventilação leva a pneumonias, atelectasias e insuficiência respiratória noturna, que se torna progressivamente diurna ${ }^{9}$. Em geral, os problemas respiratórios se agravam com os efeitos negativos sofridos pela mecânica do tórax, que se deforma na altura dos pulmões devido ao desenvolvimento progressivo da escoliose ${ }^{5}$.

Cerca de 75-90\% dos pacientes com DMD desenvolvem escoliose progressiva após a perda da marcha ${ }^{10,11}$. A progressão da deformidade é associada com o desenvolvimento de quadros dolorosos e está associada com insuficiências cardíaca e respiratória ${ }^{10,12,13}$.

Nesse contexto, há na literatura estudos que correlacionam a deformidade espinhal e a função pulmonar, como Inal-Ince et al. ${ }^{14}$ que investigaram o efeito da escoliose na função respiratória de pacientes com doença neuromuscular e encontraram evidências de comprometimento restritivo pulmonar e fluxos expiratórios reduzidos quando comparados com o grupo sem escoliose.

Kurz et al. ${ }^{15}$ analisaram a função respiratória de 25 pacientes com DMD e observaram que, a cada 1 ano, a influência negativa sobre a capacidade vital forçada (CVF) era semelhante a influência de a cada 10 graus de escoliose, sendo que em ambos ocorre uma diminuição de 4\% na CVF. Esse declínio ocorreu mais lentamente quando a progressão da escoliose foi interrompida por fusão espinal. $\mathrm{Na}$ mesma linha, os resultados de Yamashita et al. ${ }^{16}$ também indicam correlação entre o plateau da capacidade vital (PCV) e a gravidade da escoliose, sugerindo que o PCV tem valor preditivo para progressão rápida e grave das deformidades espinhais. Em contraste, há estudos ${ }^{17-19}$ em que os autores não encontraram diferenças na taxa de deterioração pulmonar quando comparados pacientes que passaram por correção da escoliose com os pacientes que não foram submetidos à fixação espinal.

A correção cirúrgica da escoliose é realizada para retardar o agravamento da deformidade e deve ser realizada antes do aparecimento de doenças cardiorrespiratórias ${ }^{10,12,13}$, mas recursos terapêuticos conservadores podem beneficiar o paciente até após a intervenção cirúrgica, resultando em melhor qualidade de vida ${ }^{20}$.

No processo de reabilitação da DMD, o terapeuta deve se concentrar na prevenção do colapso do tronco, na manutenção do equilíbrio do tronco e promover maior estabilidade da postura sentada ${ }^{21}$. Conforme a doença progride, uma cadeira de rodas com adequação postural é uma forma de retardar o desenvolvimento de deformidades, manter certo grau de mobilidade e qualidade de vida ${ }^{3,22}$. Com o objetivo de atender as especificidades de cada paciente, a adequação em cadeiras de rodas tem sido desenvolvida para promover uma postura mais satisfatória ${ }^{23}$. No entanto, o efeito dessas adaptações na função respiratória precisa de análise mais aprofundada. O objetivo do presente estudo foi avaliar a possível influência da adequação postural em cadeira de rodas em volumes, capacidades pulmonares e pressões respiratórias máximas estáticas em pacientes com DMD cadeirantes. 


\section{METODOLOGIA}

O estudo foi aprovado pelo Comitê de Ética em Pesquisa. Participaram do estudo 12 pacientes com diagnóstico de DMD, com idade média de 15,0 $\pm 3,9$ (variando 10-22) anos, que possuíam cadeira de rodas com adequação postural efetuada no máximo um ano antes da data da avaliação. Os critérios de inclusão foram o diagnóstico confirmado por meio de análise genética, possuir prova de função pulmonar, capacidade de cooperar com a avaliação da função pulmonar e consentimento dos responsáveis. Os critérios de exclusão foram instabilidade clínica, infecção respiratória aguda dentro de duas semanas precedentes da avaliação, traqueostomia e fixação da coluna vertebral.

A avaliação foi feita em uma sessão e constituiu-se em duas fases: (1) paciente em sua cadeira de rodas com adequação postural e (2) em uma cadeira de rodas sem adequação postural, reclinação ou tilt. Quanto às adaptações, podiam incluir, de acordo com a necessidade de cada paciente, apoio para a cabeça, tronco, braços e pés, além de assento e encosto em espuma sobre uma base rígida, confeccionados especificamente para cada paciente visando mobilidade, autonomia, conforto, alívio de pressão e facilidades, promovendo suporte para o corpo. Essas adaptações podiam ser confeccionadas manualmente ou, em alguns casos, esculpidas de forma digitalizada de forma a promover o equilíbrio entre o melhor alinhamento biomecânico possível, o conforto e a maximização das funções fisiológicas.

$\mathrm{O}$ volume minuto (VM), volume corrente (VC) e CVF foram obtidos por meio da ventilometria, utilizando-se um ventilômetro (Ferraris Respirometer ${ }^{\mathrm{TM}}$, Wright MK) acoplado na boca do paciente por meio de um bucal e com oclusão nasal. O paciente foi orientado a respirar tranquilamente durante um minuto no ventilômetro e, dessa forma, o VM foi obtido em $\mathrm{mL} / \mathrm{min}$. O $\mathrm{VC}$ foi determinado pela divisão do VM pela frequência respiratória obtida. A CVF foi obtida em $\mathrm{mL}$, por meio de uma inspiração plena seguida de uma expiração máxima forçada. As pressões inspiratória e expiratória máximas (PImáx e PEmáx) foram mensuradas ao nível da boca por meio de manovacuômetro (Instrumentation Industries $^{\mathrm{TM}}$, Inc NS 120-TRR) e os valores expressos em $\mathrm{cmH}_{2} \mathrm{O}$. A PImax foi mensurada a partir do volume residual e a PEmax da capacidade pulmonar total, mantidas por 3 s. O pico de fluxo expiratório (PFE) foi quantificado por meio de um medidor de fluxo expiratório mini-Wright, com escala variando de 30 a $400 \mathrm{~L} / \mathrm{min}$. O teste foi efetuado pela realização de uma inspiração máxima seguida por uma expiração máxima forçada, curta e explosiva, feita no medidor. Para ser considerado o esforço, foi necessária a duração de no mínimo 1 a $2 \mathrm{~s}^{24}$.

Os dados foram inicialmente analisados quanto à aderência à curva de Gauss, pelo teste de KomogorovSimonov. As variáveis analisadas apresentaram-se paramétricas e foram expressas por meio de média e desvio padrão. Para comparação entre as médias dos resultados na cadeira de rodas sem e com adequação postural foi utilizado o teste $t$ dependente. Os valores de $\mathrm{p}<0,05$ foram considerados significantes. Os dados foram organizados e tabulados utilizando-se o programa Statistical Package for the Social Sciences (SPSS) v.19.0.

\section{RESULTADOS}

Foram triados 134 pacientes acompanhados na Instituição, 43 (32\%) ainda deambulavam, 22 (16,4\%) haviam sido submetidos à fixação da coluna vertebral, 13 (9,7 \%) não dispunham de transporte para chegar à Instituição, $26(19,4 \%)$ não possuíam cadeiras de rodas com adequação postural, $18(13,4 \%)$ possuíam cadeiras de rodas que necessitavam de revisão (tamanho e reparação das adaptações), e 12 (8,9\%) pacientes possuíam cadeiras de rodas com adequação postural adequada e assim participaram do estudo.

Os participantes perderam a capacidade de andar com $9,4 \pm 1,5$ anos e obtiveram sua primeira cadeira de rodas com adequação postural em média de $12,4 \pm 3,0$ anos de idade. Os pacientes apresentam escoliose com ângulo de Cobb superior a $60^{\circ}$ (média $87,0 \pm 14,8$ ). Permaneciam em média 8,1 $\pm 4,5$ (variando 2-14) h/dia na cadeira de rodas.

Os valores dos volumes e capacidade pulmonar, pressões respiratórias máximas estáticas e PFE foram maiores e estatisticamente significativos quando mensurados com os pacientes em suas cadeiras de rodas com adequação postural (Tabelas 1 e 2).

\section{DISCUSSÃO}

A principal função da adequação postural em cadeiras de rodas é promover o alinhamento mais adequado do corpo, evitando compensações da coluna vertebral. Considerando que o melhor alinhamento beneficia 
Tabela 1. Valores encontrados para volume minuto, volume corrente e capacidade vital forçada dos pacientes com distrofia muscular de Duchenne em cadeira de rodas sem adequação postural e com adequação postural

\begin{tabular}{|c|c|c|c|c|c|c|}
\hline \multirow[b]{2}{*}{ CASOS } & \multicolumn{2}{|c|}{ Volume minuto (mL/min) } & \multicolumn{2}{|c|}{ Volume corrente (mL) } & \multicolumn{2}{|c|}{ Capacidade vital forçada (mL) } \\
\hline & SAP & AP & SAP & AP & SAP & AP \\
\hline 1 & 5.600 & 11.650 & 350 & 448 & 466 & 1.200 \\
\hline 2 & 5.950 & 9.900 & 160 & 412 & 650 & 1.000 \\
\hline 3 & 10.200 & 12.750 & 283 & 455 & 850 & 1.600 \\
\hline 4 & 20.000 & 19.500 & 550 & 475 & 3.100 & 3.200 \\
\hline 5 & 5.060 & 5.150 & 316 & 400 & 2.750 & 2.750 \\
\hline 6 & 7.300 & 7.500 & 260 & 312 & 1.950 & 2.000 \\
\hline 7 & 5.800 & 9.100 & 215 & 415 & 1.000 & 1.300 \\
\hline 8 & 6.700 & 10.900 & 257 & 545 & 1.100 & 1.700 \\
\hline 9 & 4.200 & 6.300 & 175 & 273 & 400 & 500 \\
\hline 10 & 6.100 & 6.550 & 234 & 344 & 1.550 & 1.500 \\
\hline 11 & 13.750 & 12.650 & 404 & 486 & 2.900 & 3.850 \\
\hline 12 & 16.900 & 17.200 & 625 & 637 & 1.000 & 1.600 \\
\hline Média & $8.963,3$ & $10.762,5$ & 319,0 & 433,5 & $1.476,3$ & $1.850,0$ \\
\hline \multirow[t]{2}{*}{ Desvio padrão } & $5.166,7$ & $4.363,2$ & 144,9 & 99,7 & 970,7 & 962,7 \\
\hline & \multicolumn{2}{|c|}{$p=0,017$} & \multicolumn{2}{|c|}{$p=0,002$} & \multicolumn{2}{|c|}{$p=0,003$} \\
\hline
\end{tabular}

SAP: cadeira de rodas sem adequação postural; AP: cadeira de rodas com adequação postural

Tabela 2. Valores encontrados para pressão inspiratória máxima, pressão expiratória máxima e pico de fluxo expiratório em pacientes com distrofia muscular de Duchenne em cadeira de rodas sem adequação postural e com adequação postural

\begin{tabular}{|c|c|c|c|c|c|c|}
\hline \multirow[b]{2}{*}{ CASOS } & \multicolumn{2}{|c|}{ Pressão inspiratória máxima $\left(\mathrm{cmH}_{2} \mathrm{O}\right)$} & \multicolumn{2}{|c|}{ Pressão expiratória máxima $\left(\mathrm{cmH}_{2} \mathrm{O}\right)$} & \multicolumn{2}{|c|}{ Pico de fluxo expiratório (L/min) } \\
\hline & SAP & AP & SAP & AP & SAP & AP \\
\hline 1 & -20 & -30 & 20 & 25 & 80 & 120 \\
\hline 2 & -25 & -45 & 20 & 35 & 80 & 150 \\
\hline 3 & -20 & -35 & 20 & 25 & 150 & 180 \\
\hline 4 & -100 & -100 & 70 & 75 & 250 & 250 \\
\hline 5 & -30 & -35 & 30 & 35 & 180 & 200 \\
\hline 6 & -100 & -85 & 35 & 40 & 170 & 160 \\
\hline 7 & -25 & -40 & 15 & 30 & 210 & 250 \\
\hline 8 & -40 & -55 & 35 & 45 & 135 & 200 \\
\hline 9 & -30 & -35 & 15 & 15 & 90 & 110 \\
\hline 10 & -35 & -70 & 40 & 45 & 200 & 180 \\
\hline 11 & -40 & -45 & 35 & 35 & 250 & 250 \\
\hline 12 & -30 & -40 & 20 & 35 & 150 & 170 \\
\hline Média & -41.2 & -51.2 & 29.6 & 36.7 & 162.1 & 185,0 \\
\hline \multirow[t]{2}{*}{ Desvio padrão } & -28.2 & -22.3 & 15.4 & 14.8 & 59.7 & 47.8 \\
\hline & \multicolumn{2}{|c|}{$p=0,015$} & \multicolumn{2}{|c|}{$p=0,001$} & \multicolumn{2}{|c|}{$p=0,16$} \\
\hline
\end{tabular}

SAP: cadeira de rodas sem adequação postural; AP: cadeira de rodas com adequação postural

a função respiratória, um estudo prévio realizado por nosso grupo ${ }^{24}$, utilizando o mesmo método de avaliação, investigou a influência da adequação postural na função respiratória de 12 pacientes com amiotrofia espinal progressiva tipo II. O resultado demonstrou diferenças estatisticamente significativas de todos os parâmetros respiratórios nas medidas tomadas na cadeira adapta$\mathrm{da}$, quando comparados aos valores com a postura adequada. Comparando-se as duas situações, o sistema de adequação postural resultou em um aumento de $16 \%$ no VM, 30\% no VC, 25\% na CVF, 46\% na PImáx, 37\% na PEmáx e 19\% no PFE.
Nessa linha, o presente estudo procurou avaliar se o mesmo ocorre em pacientes com DMD quando dependentes de cadeira de rodas. Nossos resultados mostraram que as adaptações também beneficiam esses pacientes, pois os valores encontrados foram diferentes e estatisticamente significativos para todos os parâmetros. Comparando-se as duas situações, o sistema de adequação postural resultou em um aumento de $25 \%$ na CVF, 20\% no VM, 36\% na VC, 24\% na PImax e PEmax, e $14 \%$ no PFE.

Poucos estudos sobre esse tema em doenças neuromusculares foram encontrados na literatura. Apesar 
de avaliarem parâmetros distintos, outros dois estudos foram realizados para avaliar o efeito da adequação postural na função respiratória de crianças com paralisia cerebral ${ }^{25}$ e pacientes com $\mathrm{DMD}^{26}$, demonstrando resultados coerentes com o do presente estudo. No estudo de Nwaobi e Smith ${ }^{26}$, o VC, volume expiratório forçado em um segundo (VEF1) e tempo expiratório (TE) de oito crianças com paralisia cerebral, com idades entre 5 e 12 anos, foram mensurados em uma cadeira de rodas simples e com sistema de adequação postural. Os resultados demonstraram um aumento de $57,7 \%$ do VC, de $51,6 \%$ do VEF1 e de $55 \%$ do TE no sistema de adequação postural, quando comparado com a cadeira de rodas simples ${ }^{8}$. De forma semelhante, Olunwa e Nwaobi ${ }^{27}$ avaliaram 8 pacientes com DMD, com idade entre 12 e 21 anos e demonstraram um aumento de $28 \%$ da CVF, de $14,5 \%$ do VEF1 e de $17,3 \%$ do TE forçado na cadeira com adaptações.

Nossos dados demonstraram que o negligenciamento em manter um alinhamento adequado do corpo, prevenindo compensações da coluna vertebral, geralmente resulta em diminuição significativa da função respiratória para pacientes com DMD dependentes de cadeira de rodas. Nesses casos, as adaptações confeccionadas especificamente para o paciente em sua cadeira de rodas forneceram melhor posicionamento e estabilização do tronco, diferentemente das cadeiras padrão sem adequação postural.

Importantes limitações do presente estudo referem-se ao tamanho da amostra, distribuição não aleatória e ao não mascaramento do avaliador. A amostra não foi baseada em um cálculo amostral específico. Tratou-se de uma amostra de conveniência da instituição. $\mathrm{O}$ avaliador não foi mascarado no estudo, pois as avaliações foram realizadas por um mesmo avaliador nos momentos com e sem adequação postural em cadeira de rodas. No entanto, o avaliador não tinha conhecimento do objetivo do estudo. Novos estudos devem ser realizados para contribuir com os presentes resultados, principalmente considerando uma amostra com tamanho adequado, com distribuição aleatória dos participantes e com avaliador cego.

\section{CONCLUSÃO}

Concluimos que a adequação postural na cadeira de rodas pode influenciar positivamente a função respiratória de pacientes com DMD. No entanto, devido à progressão da doença, é necessária a avaliação periódica da função pulmonar e da progressão da escoliose, a fim de se identificar a melhor alternativa terapêutica em cada fase.

\section{REFERÊNCIAS}

1. Eagle M, Bourke J, Bullock D, Gibson M, Mehta J, Gidding D, et al. Managing Duchenne muscular dystrophy -- the additive effect of spinal surgery and home nocturnal ventilation in improving survival. Neuromuscul Disord. 2007;17(6):470-5.

2. Balaban B, Matthews DJ, Clayton GH, Carry T. Corticosteroid treatment and functional improvement in Duchenne muscular dystrophy: Long-term effect. Am J Phys Med Rehabil. 2005;84(11):843-50.

3. Brunherotti MA, Sobreira C, Rodrigues-Júnior AL, Assis MR, Terra Filho J, Martinez AB. Correlations of Egen Klassifikation and Barthel Index scores with pulmonary function parameters in Duchenne muscular dystrophy. Heart Lung. 2007;36(2):132-9.

4. Tangsrud SE, Petersen IL, Lodrup Carlsen KC, Carlsen KH. Lung function in children with Duchenne's muscular dystrophy. Respir Med. 2001;95(11):898-903.

5. Velasco MV, Colin AA, Zurakowski D, Darras BT, Shapiro F. Posterior spinal fusion for scoliosis in Duchenne muscular dystrophy diminishes the rate of respiratory decline. Spine 2007;32(4):459-65.

6. Bushbya K, Bourkeb J, Bullockc R, Eaglea M, Gibsond M, Quinbyd J. The multidisciplinary management of Duchenne muscular dystrophy. Curr Paediatr. 2005;15(4):292-300.

7. Hahn A, Bach JR, Delaubier A, Renardel-Irani A, Guillou C, Rideau Y. Clinical implications of maximal respiratory pressure determinations for individuals with Duchenne muscular dystrophy. Arch Phys Med Rehabil. 1997;78(1):1-6.

8. Koessler W, Wanke T, Winkler G, Nader A, Toifl K, Kurz H, et al. 2 years' experience with inspiratory muscle training in patients with neuromuscular disorders. Chest. 2001;120(3):765-9.

9. American Thoracic Society Documents. Respiratory care of the patient with Duchenne Muscular Dystrophy. ATS Consensus Statement. Am J Respir Crit Care Med. 2004;170(4):456-65.

10. Arun R, Srinivas S, Mehdian SM. Scoliosis in Duchenne's muscular dystrophy: a changing trend in surgical management: a historical surgical outcome study comparing sublaminar, hybrid and pedicle screw instrumentation systems. Eur Spine J. 2010;19(3):376-83.

11. Kinali M, Messina S, Mercuri E, Lehovsky J, Edge G, Manzur AY, et al. Management of scoliosis in Duchenne muscular dystrophy: a large 10-year retrospective study. Dev Med Child Neurol. 2006;48(6):513-8.

12. Gayet LE, Duport G, Pries P. Flexible and semi-early vertebral instrumentation in surgical treatment of Duchenne muscular dystrophy scoliosis. Eur J Orthop Surg Traumatol. 1999;9(4):223-31.

13. Mehta KS, Gibson MJ. The treatment of neuromuscular scoliosis. Curr Orthop. 2003;17(4):313-21.

14. nal-Ince D, Savci S, Arikan H, Saglam M, Vardar-Yagli N, Bosnak-Guclu M, et al. Effects of scoliosis on respiratory muscle strength in patients with neuromuscular disorders. Spine J. 2009;9(12):981-6.

15. Kurz LT, Mubarak SJ, Schultz P, Park SM, Leach J. Correlation of scoliosis and pulmonary function in Duchenne muscular dystrophy. J Pediatr Orthop. 1983;3(3):347-53. 
16. Yamashita T, Kanaya K, Yokogushi K, Ishikawa Y, Minami R. Correlation between progression of spinal deformity and pulmonary function in Duchenne muscular dystrophy. J Pediatr Orthop. 2001;21(1): 113-6.

17. Kennedy JD, Staples AJ, Brook PD, Parsons DW, Sutherland AD, Martin AJ, et al. Effect of spinal surgery on lung function in Duchenne muscular dystrophy. Thorax. 1995;50(11):1173-8.

18. Miller RG, Chalmers AC, Dao H, Filler-Katz A, Holman D, Bost F. The effect of spine fusion on respiratory function in Duchenne muscular dystrophy. Neurology. 1991;41(1):38-40.

19. Miller F, Moseley CF, Koreska J, Levison H. Pulmonary function and scoliosis in Duchenne dystrophy. Pediatr Orthop. 1988;8(2): 133-7.

20. Cervellati S, Bettini N, Moscato M, Gusella A, Dema E, Maresi R. Surgical treatment of spinal deformities in Duchenne muscular dystrophy: a long term follow-up study. Eur Spine J. 2004:13(5):441-8.

21. Kotwicki T, Jozwiak M. Conservative management of neuromuscular scoliosis: Personal experience and review of literature. Disabil Rehabil. 2008;30(10):792-8.
22. Liu M, Mineo K, Hanayama K, Fujiwara T, Chino N. Practical problems and management of seating through the clinical stages of Duchenne's muscular dystrophy. Arch Phys Med Rehabil. 2003;84(6):818-24.

23. Hatta T, Nishimura S, Inoue K, Yamanaka M, Maki M, Kobayashi N, et al. Evaluating the relationship between the postural adaptation of patients with profound cerebral palsy and the configuration of the seating buggy's seating support surface. J Physiol Anthropol. 2007:26(2):217-24.

24. Pereira CAC. Diretrizes para testes de função pulmonar - Espirometria. J Pneumol. 2002;28(3):S1-S81.

25. Collange LA, Rodini C, Juliano Y, Misao MH, Isola AM, Almeida SB. Influence of wheelchair positioning aids on the respiratory function of patients with type II spinal muscular atrophy. Fisioter Pesq. 2009;16(3):229-32.

26. Nwaobi OM, Smith PD. Effect of adaptive seating on pulmonary function of children with cerebral palsy. Dev Med Child Neurol. 1986;28(3):351-4

27. Olunwa M, Nwaobi OM. Adaptive seating and pulmonary function in adults with muscular disease. Clin Rehabil. 1987;1(4):283-6. 